\title{
Isotope engineering in carbon nanotube systems
}

\author{
F. Simon, Ch. Kramberger, R. Pfeiffer, and H. Kuzmany \\ Institut für Materialphysik, Universität Wien, Strudlhofgasse 4, A-1090 Wien, Austria \\ V. Zólyomi and J. Kürti \\ Department of Biological Physics, Eötvös University, Budapest, Hungary \\ P. M. Singer and H. Alloul \\ Laboratoire de Physique des Solides, UMR 8502, \\ Université Paris-Sud, Bâtiment 510, 91405 Orsay, France
}

\begin{abstract}
We report on single-wall carbon nanotube (SWCNT) specific ${ }^{13} \mathrm{C}$ isotope enrichment. The high temperature annealing of isotope enriched fullerenes encapsulated in SWCNTs yields double-wall carbon nanotubes (DWCNTs) with a high isotope enrichment of the inner wall. The vibrational spectra evidences that no carbon exchange occurs between the two walls. The method facilitates the identification of the Raman signal of the outer and inner tubes. Nuclear magnetic resonance proves the significant contrast of the isotope enriched SWCNTs as compared to other carbon phases, and provides information on the electronic properties of the small diameter inner tubes of the DWCNTs.
\end{abstract}

Isotope engineering (IE) of materials provides an important degree of freedom for both fundamental studies and applications. The change in phonon energies upon isotope substitution, while leaving the electronic properties unaffected, have given insight into underlying fundamental mechanisms, such as phonon-mediated superconductivity [1]. Applications of IE involve for instance the controlled doping of isotope engineered ready-prepared Si heterostructures by means of neutron irradiation [2], fabrication of mononuclear devices with controlled heat conducting properties [3] , and the basic architecture for spintronics and quantum computing [4]. Recently, singlewall carbon nanotubes (SWCNTs) have been intensively studied as a result of the envisaged broad range of applicability and their unique physical properties related to their quasi one-dimensional electronic structure. Examples include the presence of Van Hove singularities in the electronic density of states [5], and the TomonagaLuttinger liquid behavior [6]. IE of carbon nanotubes using isotope enriched graphite as the starting material was attempted in order to allow NMR spectroscopy [7, [8]. However, the NMR studies have been hampered by the fact that the ${ }^{13} \mathrm{C}$ NMR active nuclei can be found in all species of carbons inevitably present in the purified SWCNT materials, and no nanotube selective enrichment or purification could be achieved until now. Vibrational spectroscopic methods are appropriate choices to study the effect of IE. For SWCNTs, Raman spectroscopy has proven to be the most convenient to characterize their electronic and structural properties. In addition, Raman studies on double-wall carbon nanotubes (DWCNTs) [9], synthesized from fullerene peapods [10], provides a unique opportunity to study the small diameter inner tubes, while the outer ones are left intact during the synthesis.

In this Letter, we demonstrate that the combination of isotope engineered DWCNTs and Raman spectroscopy can give an unprecedented opportunity to identify the as yet unobserved vibrational modes, such as the D-modes of very small diameter SWCNTs. We achieved this by developing a ${ }^{13} \mathrm{C}$ isotope enrichment method of SWCNTs specific to nanotubes. We first synthesized SWCNTs encapsulating ${ }^{13} \mathrm{C}$ enriched $\mathrm{C}_{60}$ and $\mathrm{C}_{70}$ fullerene molecules, then a high temperature treatment transformed the fullerenes into a second smaller diameter inner tube that is isotope enriched, which reflects the enrichment level of the fullerenes. A significant contrast with respect to the non-enriched carbon phases is achieved, as proven by NMR spectroscopy, and allows the direct study of very small diameter SWCNTs.

Commercial SWCNT material (Nanocarblab, Moscow, Russia [1]), ${ }^{13} \mathrm{C}$ isotope enriched fullerenes (MER Corp., Tucson, USA), and fullerenes of natural carbon (Hoechst AG, Frankfurt, Germany) were used to prepare fullerene peapods $\mathrm{C}_{60}, \mathrm{C}_{70} @ \mathrm{SWCNT}$. The SWCNTs were purified by the supplier to $50 \%$. The tube diameter distribution was determined from Raman spectroscopy [12] and we obtained $d=1.40 \mathrm{~nm}$ and $\sigma=0.10 \mathrm{~nm}$ for the mean diameter and the variance of the Gaussian distribution, respectively. We used two supplier provided grades of ${ }^{13} \mathrm{C}$ enriched fullerenes: 25 and $89 \%$ (whose values are slightly refined in our study). The $25 \%$ and the $89 \%$ grades were both composed of $\mathrm{C}_{60} / \mathrm{C}_{70} /$ higher fullerene mixtures in relative proportions of $75 \%: 20 \%: 5 \%$ (de-

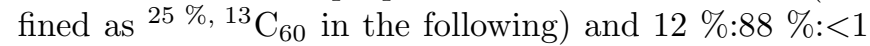

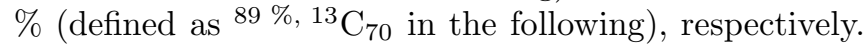
The natural carbon $\mathrm{C}_{60}\left({ }^{\mathrm{Nat}} \mathrm{C}_{60}\right)$ had a purity of $>99$ $\%$. The SWCNTs and the fullerenes were sealed under vacuum in a quartz ampoule and annealed at 650 ${ }^{\circ} \mathrm{C}$ for 2 hours for the fullerene encapsulation [13]. The peapods were transformed to DWCNTs by a dynamic vacuum treatment at $1250{ }^{\circ} \mathrm{C}$ for 2 hours following Ref. 9]. Vibrational analysis was performed on a Dilor xy triple Raman spectrometer in the 1.64-2.54 eV (676-488 


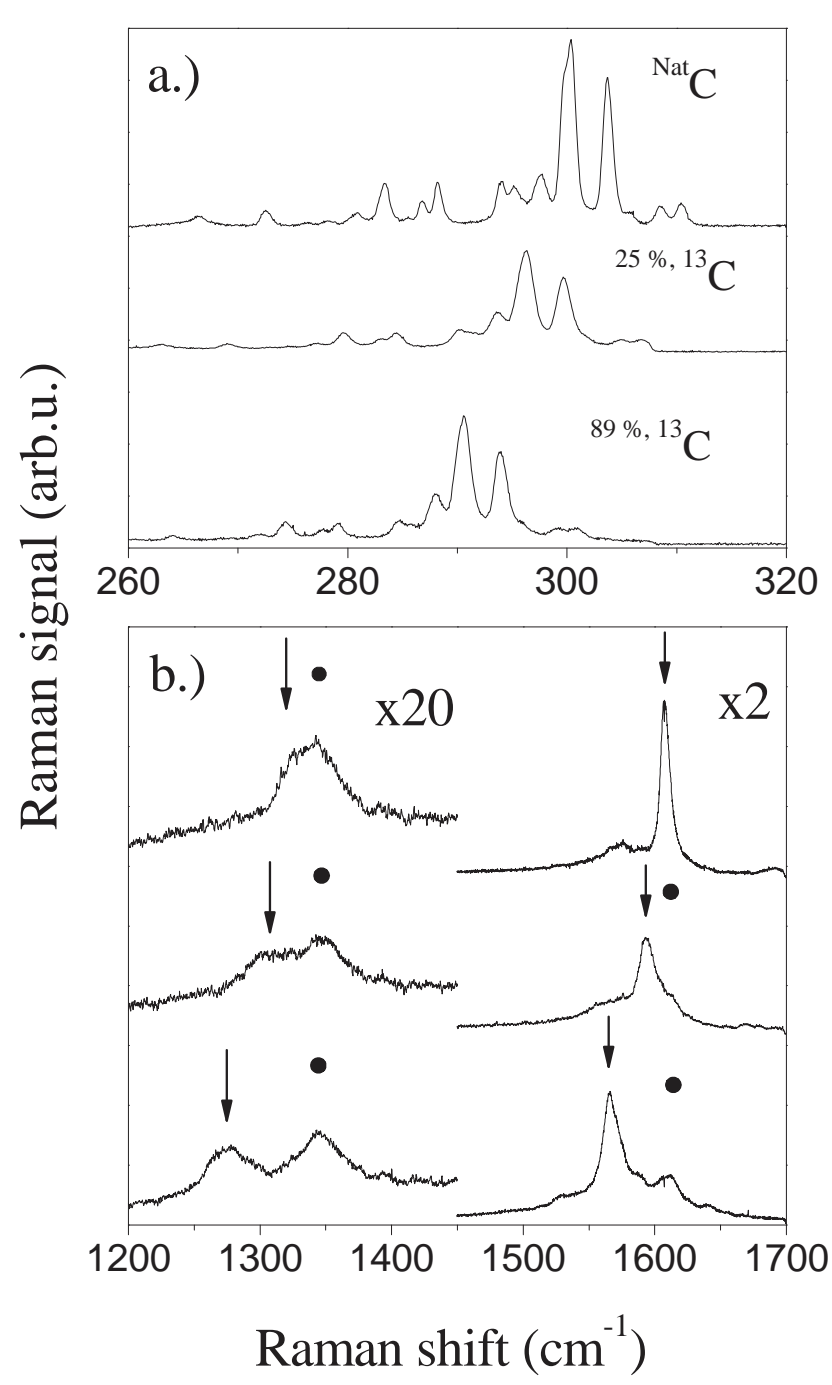

FIG. 1: Raman spectra of DWCNTs with ${ }^{N a t} \mathrm{C}$ and ${ }^{13} \mathrm{C}$ enriched inner tubes at $\lambda=676 \mathrm{~nm}$ laser excitation and $90 \mathrm{~K}$. a.) RBM Raman response b.) D and G mode Raman response. Arrows and filled circles indicate the $\mathrm{D}$ and $\mathrm{G}$ modes corresponding to the inner and outer tubes, respectively.

$\mathrm{nm}$ ) energy range at $90 \mathrm{~K}$. The spectral resolution was $0.5-2 \mathrm{~cm}^{-1}$ depending on the laser wavelength and the resolution mode used (high or normal resolution). Ab initio calculations were performed with the Vienna ab initio Simulation Package (VASP) 14]. Magic angle spinning (MAS) and static ${ }^{13} \mathrm{C}-\mathrm{NMR}$ spectra were measured in air at room temperature using a Chemagnets (Varian Inc.) MAS probe at 7.5 Tesla. The ${ }^{13} \mathrm{C}-\mathrm{NMR}$ spectra were obtained by a Fourier transformation of the free induction decay following the excitation pulse.

In Fig. 1 we show the Raman spectra of DWCNTs

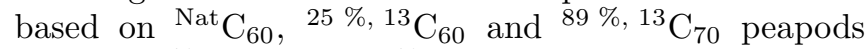
( ${ }^{\text {Nat }} \mathrm{C}-,{ }^{25 \%, 13} \mathrm{C}$ - and ${ }^{89 \%, 13} \mathrm{C}$-DWCNT) for the radial breathing mode (RBM) (Fig. Th), and the D and G mode spectral ranges (Fig. 1b) at $676 \mathrm{~nm}$ excitation. The nar- row lines in Fig. 17 were previously identified as the RBMs of the inner tubes 15. An overall downshift of the inner tube RBMs is observed for the ${ }^{13} \mathrm{C}$ enriched materials accompanied by a broadening of the line. The downshift is clear evidence for the effective ${ }^{13} \mathrm{C}$ enrichment of inner tubes. The magnitude of the enrichment and the origin of the broadening are discussed below.

The RBM lines are well separated for inner and outer tubes due to the $\nu_{\mathrm{RBM}} \propto 1 / d$ relation, where $d$ is the tube diameter. However, other vibrational modes such as the defect induced D and the tangential G modes strongly overlap for inner and outer tubes. Arrows in Fig. 10 indicate a gradually downshifting component of the observed D and G modes. This component is assigned to the $\mathrm{D}$ and $\mathrm{G}$ modes of the inner tubes. The sharper appearance of the inner tube $\mathrm{G}$ mode, as compared to the outer components, is related to the excitation of semiconducting inner tubes and the Fano line-shape broadened metallic outer tubes 15, 16.

The relative magnitude of the inner to outer tube $\mathrm{D}$ modes are comparable, as best seen for the highest enriched sample. The D mode originates from a double resonance process, which is induced by the defects in the sample [17, 18, 19]. The inner tubes were shown to contain significantly less defects than the outer ones, as proven by the narrow RBM phonon line-widths [15]. Thus, the experimentally observed $\mathrm{D}$ band intensity ratio suggests that a competitive effect, such as the enhanced electron-phonon coupling in small diameter SWCNTs [20], compensates for the smaller number of defects.

The shift of the RBM, D and G modes were analyzed for the two grades of enrichment. We found that $\left(\nu_{0}-\nu\right) / \nu_{0}=0.0109(3)$ and $0.0322(3)$ for the 25 and 89 $\%$ samples, respectively. Here, $\nu_{0}$ and $\nu$ are the Raman shifts of the same inner tube mode in the natural carbon and enriched materials, respectively. In the simplest continuum model approximation, the shift originates from the increased mass of the inner tube walls. This gives $\left(\nu_{0}-\nu\right) / \nu_{0}=1-\sqrt{\frac{12+c_{0}}{12+c}}$, where $c$ is the concentration of the ${ }^{13} \mathrm{C}$ enrichment on the inner tube, and $c_{0}=0.011$ is the natural abundance of ${ }^{13} \mathrm{C}$ in carbon. This gives $c=27.7(7)$ and $82.4(8){ }^{13} \mathrm{C}$ enrichment for the 25 and 89 $\%$ samples, respectively. The difference between the supplier provided values and those determined herein reflects the uncertainties in the enrichment level determination.

We have verified the validity of the continuum approximation for the RBM by performing first principles calculations on the $(5,5)$ tube as an example. First, the Hessian was determined by DFT calculations. Then, a large number of random ${ }^{13} \mathrm{C}$ distributions were generated and the RBM vibrational frequencies were determined from the diagonalization of the corresponding dynamical matrix for each individual distribution. We observed that the distribution of the resulting RBM frequencies can be approximated by a Gaussian whose center and variance 


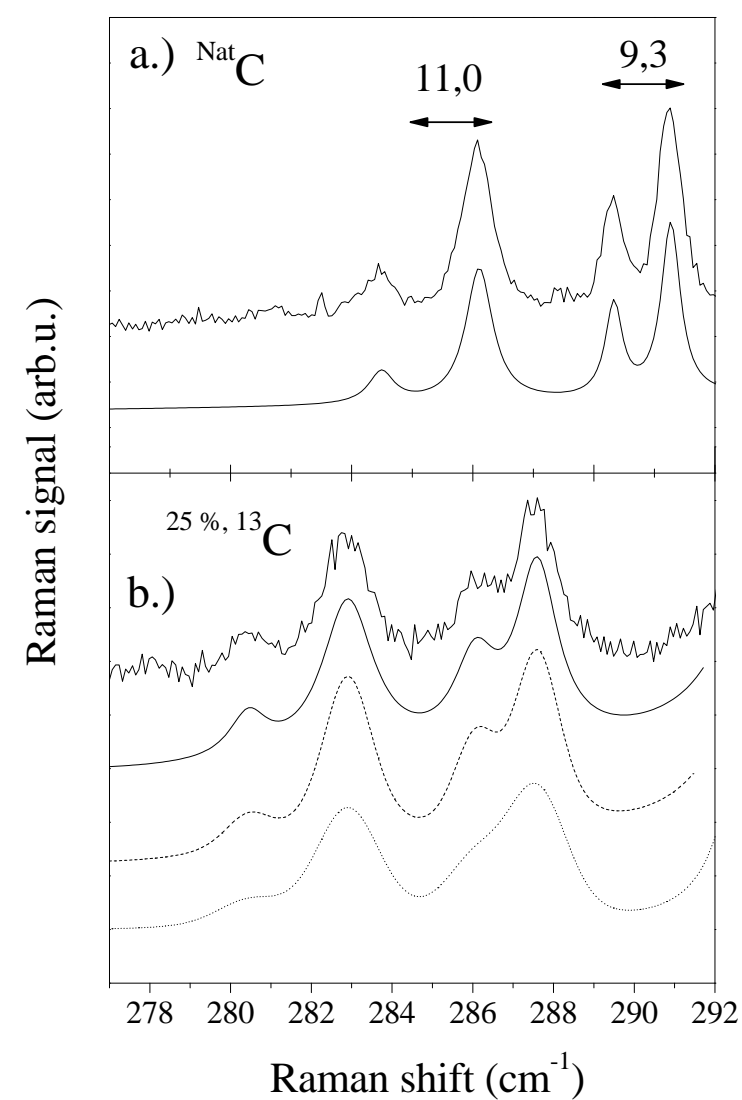

FIG. 2: RBMs of the $(11,0)$ and the $(9,3)$ inner tubes at $\lambda=676 \mathrm{~nm}$ laser excitation with $0.5 \mathrm{~cm}^{-1}$ spectral resolution. a.) ${ }^{N a t}$ C, b.) ${ }^{25 \%, 13}$ C. Smooth solid curves are the simulated Lorentzian components after deconvolution by the spectrometer response. Dashed curve is simulated line-shape with an extra Gaussian broadening to the intrinsic lines of the ${ }^{N a t} \mathrm{C}$ material. Dotted curve is calculated line-shape (see text).

determines the isotope shifted $\mathrm{RBM}, \nu$, and a spread in the RBM frequencies. We found the difference between the shift determined from the continuum model and from the $a b$ initio calculations to be below $1 \%$.

The broadening for the ${ }^{13} \mathrm{C}$ enriched inner tubes is best observed for the narrow RBMs. In Fig. 2 $\mathrm{a}$ - $\mathrm{b}$ we show the RBMs of the previously identified $(11,0)$ and $(9,3)$ inner tubes [15, 21] with their split components for the ${ }^{\mathrm{Nat}} \mathrm{C}$ and ${ }^{25 \%, 13} \mathrm{C}$ samples. Solid curves are the line-shapes after deconvolution with the Gaussian response of our spectrometer. In Fig. 2 $\mathrm{k}$, this is a Lorentzian line-shape, but in Fig. 2b, the line-shape still contains a Gaussian component, as discussed below. The FWHMs of the resulting line-shapes are 0.76(4), 0.76(4), 0.44(4), 0.54(4) and $1.28(6), 1.30(6), 1.12(6), 1.16(6)$ for the 2 components of the $(11,0)$ and $(9,3)$ inner tube RBMs of the ${ }^{\mathrm{Nat}} \mathrm{C}$ and ${ }^{25 \%, 13} \mathrm{C}$ materials, respectively. The origin of the extra broadening is due to the random distribution of ${ }^{12} \mathrm{C}$ and ${ }^{13} \mathrm{C}$ nuclei, whose magnitude was determined from the first principles calculations. We found that the

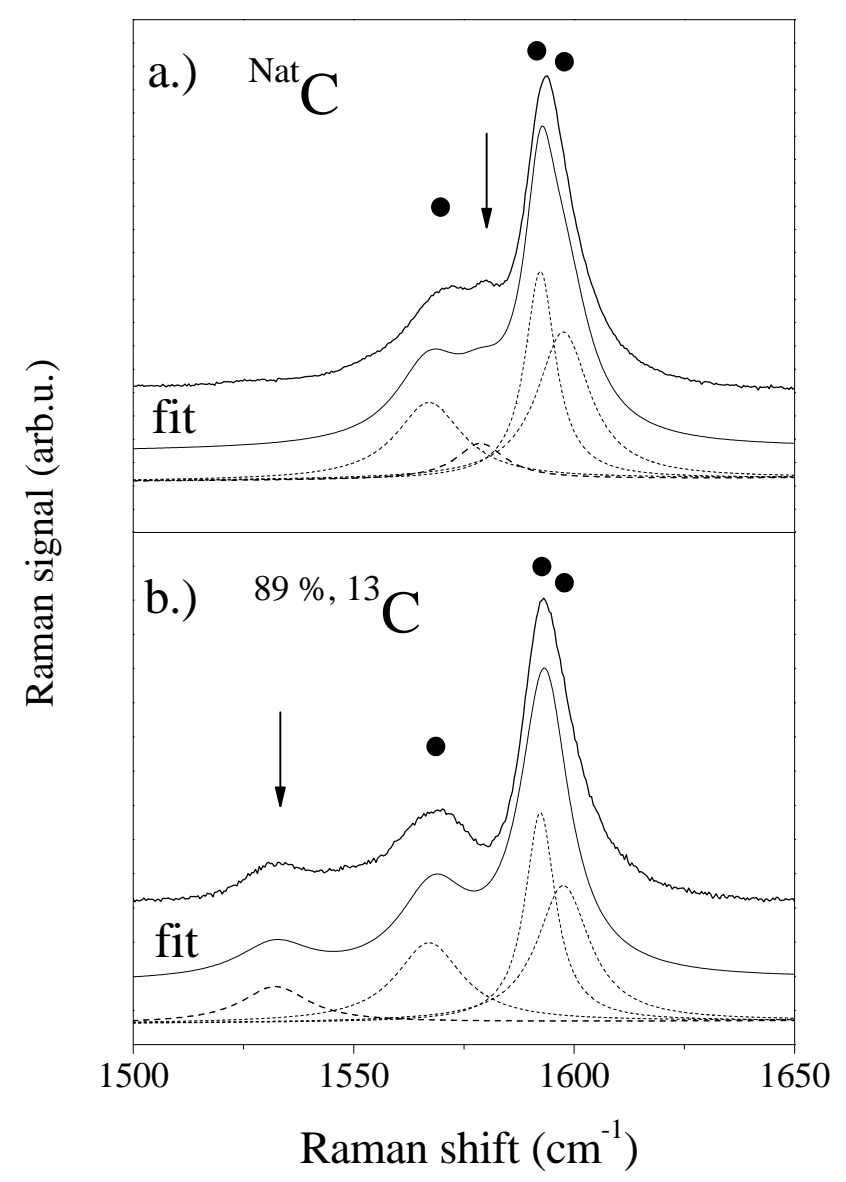

FIG. 3: G modes of DWCNT with ${ }^{\text {Nat }} \mathrm{C}$ (a) and $89 \%{ }^{13} \mathrm{C}$ (b) enriched inner walls at $\lambda=488 \mathrm{~nm}$ laser excitation and $90 \mathrm{~K}$. Filled circles indicate the non-shifting components of the outer tube $\mathrm{G}$ modes. Arrows show the only shifting componenent, identified as the inner tube $\mathrm{G}$ mode. Dashed curves show the deconvolution of the observed spectra.

ratio of the half width of extra broadening and the shift, $\Delta \nu /\left(\nu_{0}-\nu\right)$, is approximately 0.19 for a $30 \%{ }^{13} \mathrm{C}$ enriched sample. The corresponding broadened line-shapes are shown with dotted curves in Fig. 2p. When the magnitude of the Gaussian randomness related broadening was fit (shown as dashed curve in Fig 2b), we found that $\Delta \nu /\left(\nu_{0}-\nu\right)=0.15$. Similar broadening was observed for the $89 \%$ sample which can also be reproduced by the calculation.

Identification of the different signals also allowed us to address whether carbon exchange between the inner and outer tubes occurs during the DWCNT synthesis. In Fig. 3 we compare the G mode spectra of DWCNTs based on ${ }^{\text {Nat }} \mathrm{C}_{60}$ and ${ }^{89 \%,}{ }^{13} \mathrm{C}_{70}$ at $488 \mathrm{~nm}$ excitation. For this excitation, the $\mathrm{G}$ mode of the outer tubes dominates the spectrum since semiconducting outer tubes and conducting inner tubes are in resonance 16]. Indeed, the only shifting component observed is small (arrows in Fig. 3) compared to the non-shifting components (filled circles in 


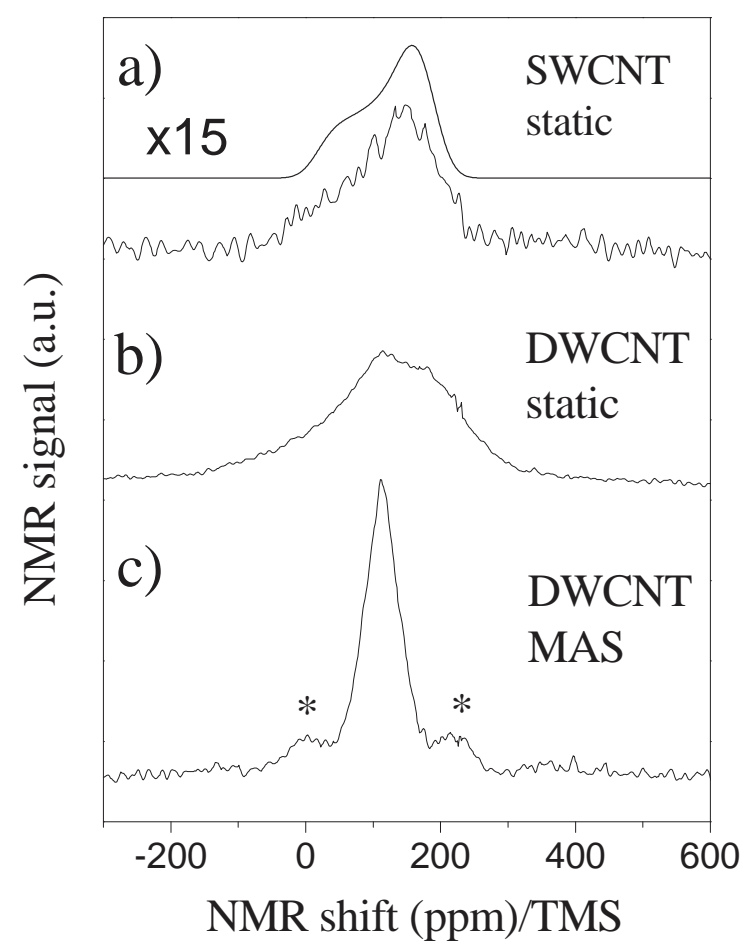

FIG. 4: NMR spectra normalized by the total sample mass, taken with respect to the tetramythilesulphate (TMS) shift. (a) Static spectrum for non-enriched SWNT enlarged by a factor 15. (b) and (c) Static and MAS spectra of DWCNT based on ${ }^{89 \%, 13} \mathrm{C}_{70}$ peapods, respectively. Asterisks show the sidebands at the $8 \mathrm{kHz}$ spinning frequency. In a) we also show a CSA powder pattern simulation with parameters published in the literature (smooth solid line) 7].

Fig. 31). It is known that the SWCNT G mode consists of several components [22, 23], however we deconvoluted the spectra with one Lorentzian line for the shifted components and three Lorentzians for the non-shifted components. We found that the spectral region above 1550 $\mathrm{cm}^{-1}$ consists of components from the outer tubes alone, that do not change upon enrichment. Separation of the inner and outer tube $\mathrm{G}$ mode components is more difficult below $1550 \mathrm{~cm}^{-1}$. We found that within the $1 \mathrm{~cm}^{-1}$ experimental accuracy, the outer tube $\mathrm{G}$ modes that are observed above $1550 \mathrm{~cm}^{-1}$ are not shifted, giving an upper limit to the extra ${ }^{13} \mathrm{C}$ in the outer wall of $1.7 \%$.

The significant ${ }^{13} \mathrm{C}$ isotope enrichment of the inner wall of the DWCNTs allowed NMR measurements with unprecedented contrast with respect to the non-enriched other carbon phases. In Fig. 廿 we show the static and MAS spectra of ${ }^{13} \mathrm{C}$ enriched DWCNTs, and the static spectrum for the SWCNT starting material. The ${ }^{13} \mathrm{C}$ signal intensity found for the enriched sample directly monitors the ${ }^{13} \mathrm{C}$ enrichment of the sample. This allowed us to determine the carbon fraction belonging to the inner tubes by comparing the total ${ }^{13} \mathrm{C}-\mathrm{NMR}$ signal intensity of the DWCNT sample and that of the ${ }^{89 \%,{ }^{13}} \mathrm{C}_{70}$ fullerene starting material. We found the mass fraction of the inner wall to be 13(4) \%, which is consistent with the 15 $\%$ inner tube mass ratio expected from the SWCNT purity $(50 \%), \sim 70 \%$ volume filling in highly filled peapod samples 24], and the mass ratio of encapsulated $\mathrm{C}_{60}$ in SWCNTs. This is the first determination of the inner tube mass content in DWCNT samples, and proves that DWCNT transformation can be achieved in macroscopic amounts.

The typical chemical shift anisotropy (CSA) powder pattern is observed for the SWCNT sample in agreement with previous reports [7, 8]. However, the DWCNT spectra can not be explained with a simple CSA powder pattern. This is the result of the larger distribution in chemical shifts since the bonding angle can show a larger variation among the small diameter inner tubes [25]. In addition, the residual line-width in the MAS experiment, which is a measure of the sample inhomogeneity, is 60(3) $\mathrm{ppm}$, i.e. larger than the $\sim 35 \mathrm{ppm}$ found previously for SWCNT samples [7, 8]. The isotropic line position, determined from the MAS measurement, is 111(2) ppm. This value is significantly smaller than the isotropic shift of the SWCNT samples, $125 \mathrm{ppm} \mathrm{7,} \mathrm{8].} \mathrm{This} \mathrm{difference}$ might originate from i.) the different $\mathrm{C}-\mathrm{C}$ bonding angle of the small inner tubes, ii.) diamagnetic shielding due to the outer tubes. Currently, experiments with inner tubes based on different diameter outer ones are underway to clarify this point.

In conclusion, we report on the synthesis of ${ }^{13} \mathrm{C}$ enriched SWCNTs. ${ }^{13} \mathrm{C}$ only resides in the SWCNTs, without enriching the inevitable other carbon phases such as amorphous carbon or graphite. The method is based on the transformation of ${ }^{13} \mathrm{C}$ enriched fullerenes encapsulating SWCNTs into DWCNTs. High levels of isotope enrichment can be achieved without significant carbon exchange between the two walls. The ${ }^{13} \mathrm{C}$ enriched inner tubes facilitate the identification of the vibrational modes of inner and outer tube components. The SWCNT selective enrichment significantly simplifies the analysis of NMR experiments where only nanotube sensitive information is desired. The synthesis method opens the way for the controllable isotope labelling of SWCNTs without labelling the unwanted side-products. The described isotope engineering may eventually find application for the controllable doping of SWCNTs, similar to the isotope engineering applied in the Si semiconducting industry.

Support from the FWF project Nr. 14893, the OTKA T038014 and the EU projects BIN2-2001-00580, MEIF-CT-2003-501099, and HPMF-CT-2002-02124 are acknowledged. Calculations were performed on the Schrödinger II cluster at the University of Vienna. 
[1] J. Bardeen, L. N. Cooper, and J. R. Schrieffer, Phys. Rev. 108, 1175 (1957).

[2] Neutron Transmutation Doping of Semiconductors, ed. by J. Meese (Plenum Press, NY, 1979).

[3] W. S. Capinski et al., Appl. Phys. Lett. 71, 2109 (1997).

[4] I. Shlimak, cond-mat/0403421

[5] M. S. Dresselhaus, G. Dresselhaus, P. C. Ecklund: Science of Fullerenes and Carbon Nanotubes, Academic Press, San Diego 1996.

[6] H. Ishii, et al., Nature 426, 540 (2003).

[7] X.-P. Tang et al., Science 288, 492 (2000).

[8] C. Goze-Bac et al., Carbon 40, 1825 (2002).

[9] S. Bandow et al., Chem. Phys. Lett. 33748 (2001).

[10] B. W. Smith et al., Nature 396, 323 (1998).
[11] http://www.nanocarblab.com

[12] H. Kuzmany et al., Eur. Phys. J. B 22 (2001) 307.

[13] H. Kataura et al., Synth. Met. 121, 1195 (2001).

[14] G. Kresse and D. Joubert, Phys. Rev. B 59, 1758 (1999).

[15] R. Pfeiffer et al., Phys. Rev. Lett. 90225501 (2003).

[16] F. Simon et al., cond-mat/0404110

[17] C. Thomsen and S. Reich, Phys. Rev. Lett. 85, 5214 (2000).

[18] J. Kürti et al., Phys. Rev. B 65, 165433 (2002).

[19] V. Zólyomi et al., Phys. Rev. Lett. 90, 157401 (2003).

[20] L. X. Bendict et al., Phys. Rev. B 52, 14935 (1995).

[21] Ch. Kramberger et al., Phys. Rev. B 68, 235404 (2003).

[22] M. A. Pimenta et al., Phys. Rev. B 58, 16016 (1998).

[23] O. Dubay et al., Phys. Rev. Lett. 88, 235506 (2002).

[24] X. Liu et al., Phys. Rev. B 65045419 (2002).

[25] J. Kürti et al., New. J. Phys. 5, 125 (2003). 